\title{
Mind Matters: A New Scientific Era ${ }^{1,2}$
}

\author{
Roger D. Nelson \\ Global Consciousness Project
}

I am an optimist, the sort we call incorrigible. So for those who know me, there should be no surprise at my title and theme. There is no question in my mind that what we do in our sometimes beleaguered bailiwick is research that provides insight into what we humans are. It is a difficult domain, so we don't have complete answers, but because of the difficulty, we are led to generate really good questions. They touch on the edges and frontiers of science (and we accept with good humor that some of our friends in academia prefer the term "fringe").

What is important is that as these challenging questions are answered, even tentatively, they will expand and extend scientific understanding far beyond the effect of mere filling in the blanks and refining details of the current picture. There are big open spaces in standard models, which are amazingly difficult for ordinary scientists to see. Where is consciousness and the mental realm we all experience? Mind has, surprisingly, been an unwanted stepchild in science over extended periods and has never been accorded a proper place in psychology or any other of the sciences, even the queen of sciences, physics. Why not? I think it is, simply put, too complicated, too difficult.

We have not known how to place consciousness or mind in a sound theoretical model and so have tended to ignore the very experiences that allow us to do physics and explore psychology.

Obviously the construction of a model of the world helps us to think about it more cogently, but somehow the construction and the thinking escape us-they are too basic, perhaps, too much like the water the fish never sees. But there is the experience, and when we focus on it and try to stay with it, we generally agree that there is a problem, perhaps even a hard problem, as David Chalmers says (1995).

During the Winchester convention, we had an opportunity to listen to Max Velmans, who also sees how much we are missing. He is one of the few adventurous souls in mainstream psychology who wants to build bridges and foundations, and eventually to create an architecture for mind, linking philosophy and psychology and physics, and, yes, parapsychology in some measure (Velmans, 2000). And we will hear committed people who are career parapsychologists discuss their contributions to an ever growing and increasingly sophisticated corpus of excellent research on the subtle but important extended qualities of human consciousness. Perhaps it is not after all too optimistic to expect a new era in science,

1 [Originally published in JP, 2008, 72, 3-17.]

2 This article is a revised version of the presidential address for the 51st Annual Convention of the Parapsychological Association, held at Winchester, England, August 13-17, 2008. 
where the experiential and subjective, mind and consciousness take important roles in models that cover the territory with more generosity and accuracy.

\section{MAINSTREAM, WHY NOT?}

We find little recognition in the scientific mainstream for what we know internally is excellent work, high quality evidence that "there is some there there." Part of the reason is that we really are addressing hard problems, with much of their difficulty stemming from a deep subtlety. In parapsychology we are looking at the edges of phenomena in an ill-defined consciousness space, and thus at problems within problems-there is no theory of consciousness, much less one that can explain the extension of consciousness into realms that, while part of human experience, are so subtle that we are seldom quite sure what has happened. But for more than a century, competent people have been piling up evidence that is persuasive to any who will look at it with care and a serious, unbiased interest. The evidence from experimental parapsychology is convergent and consistent. It shows small effects and patterns that begin to look like pieces in a puzzle. When we take an overview, a general understanding looks tantalizingly near. In addition, we have some good though still metaphoric models. We can and should feel encouraged.

Our science asks for a leap from the classical thinking that still pervades the views of most scientists even in a quantum era, but we aren't alone in that. The July 2008 edition of Scientific American has an article on "metabolomics," something most of us have never encountered. It is about the work of Jeremy Nicholson, at the Imperial College London, studying the 1,000 varieties of intestinal flora. He suggests a future of preventative therapies for serious diseases with drugs targeting the bacteria that define our unique metabolic profile. He says, "Many microbiologists might argue this is fanciful, but you only make huge progress in science by thinking almost the unthinkable" (Wenner, 2008).

More to the point in which we are most interested, there is increasing likelihood that psi research, our scientific examination of "the unthinkable," will add value to both physics and biology. Here is an observation by Dean Radin, discussing new mainstream findings about room-temperature entanglement:

Does entanglement exist in biological systems? Of course it does. Quantum connections never go away. They just become more difficult to detect when dealing with squishy, hot environments. So the better question is [how we can] detect it. There the answer is still very speculative, but I suspect we're doing it all the time, and at the psychological level we call the effect of biological entanglements (an emergent property of more elementary forms of entanglement) psi.

This quote is from personal communication, but Dean's book Entangled Minds gives background and deep discussion in the context of parapsychological findings (Radin, 2006).

There is an outside chance that regular academic biologists and physicists will catch up with parapsychologists in the near future. Otherwise it looks very likely that the folks who have chosen psi research as their primary focus despite (or indeed because of) the challenges will write the opening chapters in a more comprehensive scientific view. Already there is a significant shift in at least one important field, medicine, to integrate and apply alternative perspectives. Good research that takes a page from parapsychology is 
being done on distant healing and other therapeutic modes that were until recently considered fantasy by academic medicine. These practices actually are ancient, yet they were almost entirely excluded from western, mainstream science in its early phases. But no longer. A few courageous and persistent individuals continued to work the problems of scientific research encompassing a broader perspective in pragmatic, outcome-oriented research showing that prayer and meditation and spiritual practices do contribute to maintaining good health and to healing when it is needed (Astin, 2000). Acupuncture effects and their travel through a real meridian system (which still is disputed by classic medicine) can actually be seen using sophisticated ultrasound measures and functional magnetic resonance imaging (Jones \& Bae, 2004). There are many examples of this kind, and evidently more to come.

In consciousness research the situation is similar but without the obvious pragmatics. Again there is ancient wisdom and cross-cultural information describing both the interconnection of mind and matter and the possibilities for anomalous information transfer that we study. I think we are beginning to see the shape of the new perspectives and insights that are needed to integrate physics and biology and psychology to finally include consciousness in models of the world. Mind matters, and we who are engaged in parapsychology know that. It remains only to develop a persuasive combination of clean, clear research data and sound explanatory models.

\section{PARTICIPATION, A SINE QUA NON}

Research with all the pieces in hand remains the best evidence (for the active researcher) because it combines as closely as possible the experiential and the scientific. I will give a few examples from my work at the Princeton Engineering Anomalies Research laboratory (PEAR), where for 22 years I participated in forming effective research questions, designing the protocols and the statistical analyses, collecting the data (including data generated as a participant myself), analyzing, and ultimately interpreting the results, all in cooperation with two or three or more other bright and interested people. We all knew the importance of getting it right. We had an extraordinary opportunity to do challenging research in a setting where we could invest the time necessary to do it well and to repeat experiments with variations that could inform and improve the work. We could and did look for what matters to consciousness interacting with physical systems, and we were able to learn important constraints in our efforts to capture anomalous information transfer. At PEAR we had the luxury of expertise and resources, and we used them well because we all knew it was a precious opportunity to learn something. We did not want to waste our time or that of anybody else who might look at our work. Here are some examples of what we learned.

\section{REMOTE PERCEPTION}

One focus at the PEAR lab was a long series of experiments looking at anomalous information transfer that we called "remote perception" or "precognitive remote perception" (PRP). The paradigm is similar to the free response remote viewing work developed by Targ and Puthoff at SRI in the 1970's (Targ \& Puthoff, 1977), and related as well to the ganzfeld work developed to a high point by Honorton (Bem \& Honorton, 1994; Honorton, 1990). At PEAR we focused on quantitative assessment, using a set of 30 binary questions to represent the free response-is the scene hectic or calm; is it characterized more by straight lines or curves; are there people or not? Using the resulting performance measures, we 
sought to determine what the constraints and necessary conditions were for successful remote perception. We asked whether the effect was diminished by greater distances between the agent at the scene and the percipient, and whether the scores were different for perception attempted before the target was visited or after the visit, compared with on-time viewing. We studied whether people were more successful when the target was determined by volitional selection at the appointed time by the agent at the scene or by random selection from a pool. We also explored variations of the quantification process, gradually increasing the number of descriptors in the questionnaire from two, to four, to a quasi-continuous scale with nine options. I can give here only a very brief overview of the results of this program over about two decades of work, but it is examined in detail in a recent PEAR paper (Dunne \& Jahn, 2003).

The most important outcome was a confirmation of the primary hypothesis: Percipients can acquire information about distant targets without normal sensory channels. The effect is subtle, but over hundreds of trials, the odds against chance explanations go to millions or hundreds of millions to one. The scores for precognitive and retrocognitive trials are similar to those for concurrent trials, with no evidence for regression over a range of several days. And distance also seems not to matter; the perception of targets at international distances is indistinguishable from relatively local targets. But some variations in the experimental conditions do have a clear effect. As we developed more refined scoring procedures, the ability to capture information about the distant targets seemed to decrease. The effort to provide more nuance and flexibility to our participants turned out to be not a boon but something of a boondoggle. Brenda Dunne, who led the PRP program, had misgivings about the quantification from the beginning because it shifted focus from experience to assessment. But it was a necessary experimental investigation which produced an answer that is important. The ability to "far see" is fragile, and its requirements must be respected. It cannot be forced into an arbitrary mold for the sake of the scientific question. Instead, we must shape our scientific approach to study anomalous perception without sacrificing the free movement of the mind that enables it.

This is a critical point for research on psi and consciousness in general, one that we should understand well enough to make it clear to outside observers, both proponents and skeptics. The core understanding is that we must respect the unique character of what we observe. The answers we obtain are in part determined by the questions we ask (a photon will be seen as a particle or a wave depending on the way we observe it). We cannot squeeze or stretch a subtle talent or an ephemeral phenomenon into any arbitrary form but must accommodate its native dimensions.

\section{MIND-MACHINE INTERACTION}

The second major experimental program at PEAR was mind-machine interactions, or MMI. We began with random event generator (REG) experiments asking participants, whom we called operators, to change the random output to higher or lower numbers, compared with baselines (Jahn, Dunne, Nelson, Dobyns, \& Bradish, 1997). We had an engineering mission, which was to find out whether human consciousness in special states might affect sensitive electronic equipment. Given that context, it will be no surprise that we were dedicated to precision and accuracy, and to a thorough and wide-ranging assessment. Ultimately, we created several unique experiments addressing similar questions using electronic, mechanical, hydrodynamic, and thermodynamic systems. Some of these were so beautiful as to deserve 
a place in a fine gallery or museum, but this aspect was intended to help create conditions conducive to the "impossible" tasks we set for our operators. Again, we were attempting to provide space and opportunity for creative consciousness, and support for the subtle requirements of interactions between intention and effects in the world.

All the experiments were technically sophisticated and aesthetically elegant in their design and implementation. We made a pendulum with a crystal bob on a rod of fused silica, enclosed in clear acrylic. Operators tried to change the damping rate while measurements were taken with a razor edge cutting a light beam with timing by a 50-ns clock. We made a delightful small fountain and monitored its transition from laminar to turbulent flow with photodiode arrays to see whether intention could augment or hinder the descent from order into chaos. And we built a random mechanical cascade of 9,000 plastic balls bouncing through an array of pegs into collecting bins, forming a distribution that we tried to shift to the left or right by sheer will or intention. This was a complex mechanical instrument $3 \mathrm{~m}$ tall, and it earned the ironic name "Murphy" after the famous law, but it served well to help us determine whether psi could change behavior on an arguably macroscopic scale. And there were more such explorations: a dual thermistor experiment asking for focused temperature changes, an interferometer displaying a shifting pattern of concentric interference fringes, a Crookes tube with a series of evanescent spheres formed by luminescent gas discharge, fluctuating iridescent patterns in a birefringent plastic lever arm. Suffice it to say that we covered a lot of ground in nearly three decades of the PEAR lab.

\section{FINDINGS}

A short list of major findings in the PEAR mind-matter interaction program includes many confirmations or replications of others' work. Indeed, the PEAR REG experiments were an extension of the work of Helmut Schmidt in particular (Schmidt, 1973) to provide a completely independent assessment using the best available technology and designs. The research continued for almost three decades, so there is much informative detail. The following summary points give some notion of the span and depth of the research findings:

1. There is an effect of conscious intention on the output or behavior of random systems.

2. The anomalous effect is very small but statistically significant over many replications.

3. Depending on conditions, effect size is approximately equivalent to parts per thousand.

4. Both high and low intentions yield correlated departures from expectation.

5. Baseline trials may show reduced variance, suggesting effects of a "baseline" intention.

6. Trials conducted with the operator in local and remote locations have similar effect sizes.

7. Trials conducted with the intentional effort prior to the data collection are also successful.

8. Experiments with two operators who are a bonded pair have significantly larger effects.

9. Serial position analysis shows early trials have a large effect that decreases but recovers.

10. Anomalous effects differ in magnitude and style for individual operators.

11. About $15 \%$ of unselected operators can achieve significant overall performance.

12. Effect size and style (symmetry of intentions) transfer from REG to other experiments.

13. Experiments with a wide variety of random sources show similar effect sizes. 
14. Effects appear to depend on time invested in intentional effort and may be teleological.

15. Anomalous effects depend primarily on psychological factors, not physical parameters.

\section{GROUP CONSCIOUSNESS}

In the early 1990's, as miniaturization of electronics allowed construction of small but competent physical random number sources, we developed protocols for collecting data in the field. The question was whether REGs might be affected by mere attention rather than intention, and, more generally, whether special states of consciousness might have a kind of "field" effect. A variant of the REG program was created to take data continuously and to allow marking of the beginning and end of time periods of interest. For example, we took the REG, connected to a laptop or palmtop computer, to concerts, rituals, religious ceremonies, sporting events, board meetings, and various other events that might create a state of "group consciousness." The protocol was simple: Moments or periods that we judged likely to produce coherent or resonant thoughts and emotions among the people attending the event were marked, and the data were later extracted for analysis. The prediction was for a variance increase (since there was no directional intention, either high or low deviations from expectation would indicate an anomalous effect). We looked at many kinds of events that we expected would produce group coherence, and for a control condition, we collected data in mundane contexts such as shopping centers, busy street corners, academic meetings, and so on.

These experiments were termed FieldREG studies, and over several years we accumulated more than 100 datasets from "resonant" situations and a smaller but substantial number from "mundane" locations (Nelson et al., 1996; 1998b). A number of special series were undertaken, including data collection at operas, cathedrals, and sacred sites such as temples and tombs in Egypt. In a nutshell, these experiments showed that the REG data tended to depart from expectation in those situations that were conducive to a melding of individuals into a group consciousness. We found a few categories that were especially powerful, or rather, reliable-in the FieldREG work, as in the laboratory experiments, effect sizes tend to be small, so that repetitions of essentially similar conditions are necessary to accumulate statistical significance. On the other hand, using a time-normalized yield measure (Nelson, 2006), these natural, real world situations have a somewhat larger effect size than that found in laboratory experiments. The largest or most reliable effects seem to involve ritual or some other influence that is designed to bring people to a shared state of mind. On consideration, this seems reasonable, though we had to learn by trial and error what the most conducive situations might be. We also found that the combination of collective activity with a special place could be counted on to produce structure in the random data sequence. For example, the Egypt series comprised a traveling group of people interested in ancient Egyptian spiritual practices, who intended to chant or meditate in sacred sites. That is, there was a preplanned set of resonance-producing activities in the appropriate contexts, intended as a respectful attempt to connect to the spirit of the sacred places we visited. This series is the most consistent and hence statistically robust subset of the entire FieldREG database (Nelson, 1997, Nelson, et al., 1998b). 


\section{FIELDREG FINDINGS}

What did we learn from several years and over 100 formal assessments of the FieldREG question? In the PEAR database, it is possible to make a strict meta-analytic combination across data subsets, and from that to draw robust conclusions. These are supported also by independent work (Radin, et al., 1996; Bierman, 1996). In all such research, it is necessary to use operational definitions, namely, a description of what is done to create or identify the item of interest, the group consciousness. In the experiment, we ask whether there are variance changes in data collected during these times. Given that background, a short list of findings includes:

1. Changes in REG behavior correlate with special states of group consciousness.

2. Situations conducive to resonant interaction produce increased data variance.

3. Practices designed to create group unity and coherence yield larger deviations.

4. Some venues may reliably yield decreased variance, but more study is needed.

5. Mundane or chaotic situations yield only normal random data sequences.

6. We infer that group consciousness can exist and can have anomalous effects.

7. The studies tentatively suggest an information field or "consciousness field" effect.

8. The nature of the questions we ask partially determines the experimental result.

9. The potential range of FieldREG applications is broad and invites further study.

Among the several replications of FieldREG work were some that looked at events in distant locations, and some that used multiple REGs. Notable among these were Dean Radin's examination of data from 5 devices in separated locations taken during the reading of the verdict in the O. J. Simpson trial (Radin, 1997), and Roger Nelson's collection of data from 12 REGs in Europe and the US during Princess Diana's funeral (Nelson et al., 1998a). Both of these events engaged the attention of millions of people, and both showed statistically significant departures from expectation at the most critical or poignant times. These and similar probes suggested it would be valuable to have a continuous record of REG data that could monitor the world stage for indications that special states of "global consciousness" might affect our instruments in a way similar to the effects of group consciousness.

\section{GLOBAL CONSCIOUSNESS PROJECT}

We began planning and building a world-spanning network of physical REG/RNG devices in late 1997. The architecture of the network was designed to use the Internet (which was coming to maturity at that time as a world-wide web) to transmit data from remote nodes to a central server for archiving. Here is a brief description of the technology: Custom software on continuously running computers at each node collects one trial (comprising the sum of 200 bits) each second from an REG on a serial port, stores the trials on the local disk, and transmits the data to a server in Princeton in checksummed 5-min packets. Custom software on the server stores the data in permanent archives with all data synchronized using network time protocols. The result is a continuously growing swath of parallel data sequences extending from August 1998 to the present time (Nelson, 2001; Bancel \& Nelson, 2008). The database is publicly available for download by anyone with an interest in checking our analyses or conducting original research. 
A large and comprehensive website at http://noosphere.princeton. edu provides details of the technology and methods, a complete record of the formal hypothesis testing we have done over the years, the primary results, a growing spectrum of deeper explorations of the data, and some interpretive efforts. To date, there are over 250 rigorously vetted, prespecified events in the formal series, including tragedies and celebrations, natural- and human-caused disasters, planned and spontaneous gatherings of great numbers. The primary experiment consists of formal events that are specified in a prediction registry prior to any examination of the data. Relatively few events are selected, and the formal series comprises $1.5 \%$ of the full 10-year, 15-gigabyte database. Since we are breaking new ground in psi research, there is little or no history of similar research to guide hypothesis specification. We therefore use a general hypothesis that allows the criteria for selecting events and analysis tools to be kept deliberately free:

Periods of collective emotional or attentional behavior in widely distributed populations will correlate with deviations from expectation in a global network of physical random event generators.

A series of replications (analyses of data corresponding to the individual global events) using this general approach allows us to maintain formal rigor while exploring a variety of occasions that bring people to a common focus. By accumulating subsets of event categories, we gain insight into psychological (or sociological) parameters that help determine the nature and magnitude of anomalous effects in the data. The approach allows considerable latitude in identifying events and constructing test statistics, but with a number of constraints. The events specified in our formal hypotheses all involve large numbers of people, geographical extension, an engaging emotional or attentional character, and they are expected to promote or entail mental coherence.

The GCP is an evolutionary development in psi research that differs qualitatively as well as quantitatively from prior research. The globally distributed network produces synchronized data in parallel sequences from dozens of physical random sources, allowing a class of investigations that includes interdevice correlations, measurement of momentary variance and covariance, assessment of distance and time as parameters, and quantitative research on the possibility that multiple random sources may augment or otherwise differentiate the response.

\section{GCP FINDINGS}

We have found that the anomalous effects typically take a different form from that observed in laboratory REG research. During 10 years of operation we have specified and analyzed 250 global events constituting our operationally defined moments of "global consciousness." The nature and scale of the database provide a number of unique opportunities and findings:

1. Globally shared thoughts and emotions can affect physical random systems.

2. When global events transpire, we find anomalous structure in the GCP data.

3. The average effect size is small, about 0.3 to 0.5 sigma, but conceptually replicable.

4. The odds against chance for the composite formal result are about 10 million to 1. 
5. The anomalous effects are seen in the collective behavior of the global network.

6. Deviation (or structure) is driven by excess pairwise correlation between RNGs.

7. Distribution statistics of RNGs are unperturbed, but they correlate during events.

8. Two independent, orthogonal correlation statistics respond similarly to the formal events.

9. The orthogonal measures of network correlation are also correlated with each other.

10. There is differential response of correlation statistics to categorized subsets of events.

11. Both correlation statistics exhibit a similar distance dependence, with scale $\sim 8000 \mathrm{~km}$.

12. The temporal course of correlations shows GCP effects have a time scale of 1 to 2 hours.

When we compound the statistics from the several independent findings, we compute a bottom line deviation of more than 5 sigma ( $Z>5$ and $p$ value on the order of 10-7), representing evidence for structure where there should be none in the random data. We think this carefully established anomaly relating consciousness and physical randomness bears implications for both the study of human consciousness and our understanding of the physical world.

Detailed discussion is beyond the present scope, but some comments are in order. It is essential to understand that we do not look for "spikes" in the data and then try to identify what caused them. Instead, we identify the event first and then analyze the corresponding data-we make a prediction before examining the data and then test it in the data. This process yields a replication series of proper hypothesis tests that in their aggregate constitute a test of the general hypothesis given earlier. We find that whereas we can measure deviations in data corresponding to the identified events, the database as a whole exhibits parameters consistent with statistical expectation. The significance of each of the results enumerated above, and of the composite bottom line, has been confirmed by extensive simulation using pseudo-random data and direct resampling analyses from the network database.

The discovery of two demonstrably independent statistics is important to the development of models and helps to constrain the range of possible explanations. It also helps assure that the anomalous results cannot be ascribed to data selection. The discovery that the anomalies are not simple, direct effects on individual REGs but are driven primarily by interdevice correlations is an instructive surprise. It is yet another indication of the complexity faced by psi researchers and an example of the importance of the questions asked. The range of distances over which the internode correlations are detectable is approximately $8,000 \mathrm{~km}$, and weighted regressions show a marginally significant decline in effect size over global range of separations. This indicates that although the measured effect is indeed global, it is nevertheless sensitive to the geographical extent of the network and the distribution of the events. We can ask what the implications are for the widespread, albeit still tentative, idea that psi effects are fundamentally nonlocal. Finally, temporal structure is also an important feature of the GCP data. Our operationally defined global consciousness would seem to have a "moment" of an hour or two, perhaps corresponding to the much faster time-scale of human consciousness where a sensory or emotional impression can form in a small part of a second, perhaps as little as 100 milliseconds. 


\section{CONVERGENT EVIDENCE}

There is a powerful general point to be made from the psi literature. Given that there are many experiments and observations of high quality showing anomalies in a wide range of disciplines, and independent findings pointing to effects of consciousness that are not accounted for in ordinary psychological or physical theories, we can say that there is excellent "convergent evidence" that consciousness interacts with physical reality. When there is just one opinion or one experimental observation on a phenomenon, it is difficult to make a case. But with more than 100 years of research by highly qualified scientists looking from different perspectives at the extended capacities and limitations of mind, we can consider whether their findings converge. I think they do, in no uncertain terms, despite, and indeed with the help of, criticisms that ultimately have strengthened the evidence. We have personal experience and observation of natural occurrences of psi. We have laboratory experiments on extrasensory perception, clairvoyance, psychometry, psychokinesis, and more. We have extensions of these efforts to learn something in the real world, some pragmatic and some purely experimental. Government and business have requested and gotten help from psi practitioners, sometimes with high profile public presence as in the Stargate program of remote viewing. Pertinent to our theme, such work may be regarded as applications of techniques and findings from controlled laboratory research (Dunne \& Jahn, 2003; Targ \& Puthoff, 1977). Similarly, studies of micro-psychokinesis in the laboratory have lead to field research on group consciousness attempting to confirm that special states of resonance or coherence reportedly stimulated by ritual, music, collaboration, and cooperation may have a detectable presence beyond the experiential (Nelson, et al., 1997; 1998a; 1998b).

The natural extrapolation of field research with REGs into the Global Consciousness Project is a multilevel example of convergent evidence. Not only does the GCP present an independent and completely different perspective on the question of whether mind has real presence in the world, but its application of powerful modeling and statistical techniques to search for structure in this large and complex database seeks convergent evidence internally. The result is a collection of findings that are on the one hand demonstrably independent and on the other hand complementary; they are interlocked pieces of a comprehensive picture. Again we find indicators of a real entity that is anomalous in the sense that ordinary physical models do not yet accommodate it. But this evidence converges with and extends the field studies of group consciousness and the laboratory research with individuals. The GCP results say essentially the same thing as do the results of decades of psi research in laboratories around the world, albeit in a different but very rich language. Consciousness is real. It has a role to play as a presence in the physical world. Our work as psi researchers is to go on with efforts to learn more about that presence and to make clear that the role of consciousness in the world is both real and important. In this first decade of the 21 st century, it is becoming apparent that that role is critical. 


\section{References}

ASTIN, J. A., \& HARKNESS, E. E. (2000). The efficacy of “distant healing”: A systematic review of randomized trials. Annals of Internal Medicine, 132, 903-910.

BANCEL, P. A., \& NELSON, R. D. (2008). Rigorous exploration of GCP data: Correlations, structure, implications. Journal of Scientific Exploration, 22(3), 309-333.

BEM, D. J., \& HONORTON, C. (1994). Does psi exist? Replicable evidence for an anomalous process of information transfer. Psychological Bulletin, 115, 4-18.

BIERMAN, D. J. (1996). Exploring correlations between local emotional and global emotional events and the behavior of a random number generator. Journal of Scientific Exploration, 10, 363-374.

CHALMERS, D. (1995). Facing up to the problem of consciousness. Journal of Consciousness Studies, 2, 200-219.

DUNNE, B. J., \& JAHN, R. G. (2003). Information and uncertainty in remote perception research. Journal of Scientific Exploration, 17, 207-241.

HONORTON, C., BERGER, R. E., VARVOGLIS, M. P., QUANT, M., DERR, P., SCHECHTER, E. I., ET AL. (1990). Psi communication in the ganzfeld: Experiments with an automated testing system and a comparison with a meta-analysis of earlier studies. Journal of Parapsychology, 54, 99-139.

JAHN, R. G., DUNNE, B. J., NELSON, R. D., DOBYNS, Y. H., \& BRADISH, G. J. (1997). Correlations of random binary sequences with prestated operator intention: A review of a 12-year program. Journal of Scientific Exploration, 11, 345-368.

JONES, J. P., \& BAE, Y. K. (2004). Ultrasonic visualization and stimulation of classical oriental acupuncture points. Medical Acupuncture, 15, 24-26.

NELSON, R. D. (1997). FieldREG measurements in Egypt: Resonant consciousness at sacred sites. (Technical note PEAR 97002). Princeton, NJ: Princeton Engineering Anomalies Research, School of Engineering/Applied Science, Princeton University.

NELSON, R. D. (2001). Correlation of global events with REG data: An internet-based, nonlocal anomalies experiment. Journal of Parapsychology, 65, 247-271.

NELSON, R. D. (2002). Coherent consciousness and reduced randomness: Correlations on September 11, 2001. Journal of Scientific Exploration, 6, 549-570.

NELSON, R. D. (2006). Time-normalized yield: A natural unit for effect size in anomalies experiments. Journal of Scientific Exploration, 20, 177- 200.

NELSON, R. D., \& BANCEL, P. A. (2006). Anomalous anticipatory responses in networked random data. In D. P. Sheehan (Ed.), Frontiers of time: Retrocausation-Experiment and theory. AIP Conference Proceedings No. 863, Melville, NY (pp. 260-272).

NELSON, R. D., BOESCH, H., BOLLER, E., DOBYNS, Y. H., HOUTKOOPER, J., LETTIERI, A., ET AL. (1998a). Global resonance of consciousness: Princess Diana and Mother Teresa. Electronic Journal for Anomalous Phenomena. Retrieved September 13, 2008, from http://noosphere.princeton. edu/ejap/diana/1998_1.html.

NELSON, R. D., BRADISH, G. J., DOBYNS, Y. H., DUNNE, B. J., \& JAHN, R. G. (1996). FieldREG anomalies in group situations. Journal of Scientific Exploration, 10, 111-141.

NELSON, R. D., BRADISH, G. J., DOBYNS, Y. H., DUNNE, B. J., \& JAHN, R. G. (1998b). FieldREG II: Consciousness field effects: Replications and explorations. Journal of Scientific Exploration, 12, 425-454.

RADIN, D. I., REBMAN, J. M., \& CROSS, M. P. (1996). Anomalous organization of random events by group consciousness: Two exploratory experiments. Journal of Scientific Exploration, 10, 43-168.

RADIN, D. I. (1997). The conscious universe: The scientific truth of psychic phenomena. San Francisco: HarperEdge. 
RADIN, D. I. (2006). Entangled minds: Extrasensory experiences in a quantum reality. New York: Paraview Pocket Books.

SCHMIDT, H. (1973). PK tests with a high-speed random number generator. Journal of Parapsychology, 37, 105-118.

TARG, R., \& PUTHOFF, H. (1977). Mind reach: Scientists look at psychic abilities. New York: Delacorte. VELMANS, M. (2000). Understanding consciousness. London: Routledge.

WENNER, M. (2008). Metabolomics: Going with his gut bacteria. Scientific American, Health, July 2008. 